\title{
Fatigue behaviour of selected materials under multiaxial asynchronous loadings
}

\author{
Łukasz Pejkowski ${ }^{1,1}$, Dariusz Skibicki ${ }^{1}$, and Jan Seyda ${ }^{1}$ \\ ${ }^{1}$ UTP University of Science and Technology in Bydgoszcz, Kaliskiego 7, 85-796 Bydgoszcz, Poland
}

\begin{abstract}
Four types of materials: PA38 aluminium alloy, E235 steel, E355 steel and 1.4301 austenitic steel were subjected to low-cycle multiaxial loadings. All tests were strain-controlled and typical, thin-walled, hollow specimens were used. Various synchronous and asynchronous loadings were applied. The analysis of experimental results involved: cyclic stress-strain response, fatigue life and observation of microcracks behaviour on the surfaces of fatigued specimens. Obtained results indicate that the difference in the strain components frequency of the asynchronous loadings has a significant influence on the fatigue behaviour of the materials.
\end{abstract}

\section{Introduction}

Multiaxial loadings, especially non-proportional, have a very significant impact on the fatigue behaviour of materials [1]. This term is used for loadings in case of which loading components are not in constant proportion. A characteristic feature of non-proportional loadings is a rotation of principal axes of stress and strain during loading cycle or block. Sometimes, under non-proportional loadings, a reduction of fatigue lives is observed, comparing to proportional loadings [2]. The modelling of the fatigue behaviour of materials subjected to non-proportional loading is complicated. The methods present in the literature are often limited to a narrow range of loading cases. Historically, the largest group of nonproportional loadings using which fatigue tests were conducted are out-of-phase loadings [3]. In the case of this type of loading, the parameter that determines the degree of nonproportionality is the phase shift angle. However, it is constant over time. Another interesting group of non-proportional loadings are the ones in case of which there is a difference in the frequency of components. These loadings are usually called asynchronous. Unfortunately, often only one case of asynchronous lading is used as one of many types of analysed multiaxial loadings and no special attention is given to it as it was noticed in [4]. At the same time, such loading is described by the same fatigue damage parameter as synchronous loadings. In cases where the difference in the frequency of component strains or stresses is significant, such an approach leads to doubts regarding the interpretation of the obtained results. Based on the above observations, we decided that it is advisable to conduct research in the field of asynchronous loadings, using a wide range of asynchronous loading cases as well as a few materials. The aim of this work was to present stress-strain response and resultant fatigue lives in a basic and simple form.

$1 \quad$ Corresponding author: lukasz.pejkowski@utp.edu.pl 


\section{Materials and methods}

Experiments were conducted on 4 types of materials: PA38-T6 (AW 6060-T6) aluminium alloy, solution heat-treated and artificially aged, E235 (1.0308) non-alloy quality steel, normalized, E355 (1.0580) non-alloy quality steel, normalized and 1.4301 (X5CrNi18-10, 304) austenitic stainless steel, soft annealed. Materials were purchased in the form of precise seamless pipes. Thin-walled, smooth tubular specimens were manufactured by CNC machining. The basic mechanical properties, yield stress $\sigma_{y}$, cyclic yield stress, $\sigma_{y}^{c}$, ultimate tensile strength $\sigma_{u}$, Young's modulus, $E$ and elastic Poisson's ratio of tested materials are given in Table 1 . The table includes also inner $\left(d_{i}\right)$ and outer $\left(d_{o}\right)$ diameters of specimens. The results obtained for IP and OP loadings were already partially published in [5].

Table 1. Basic mechanical properties of tested materials and dimensions of specimens.

\begin{tabular}{lccccccc}
\hline \multirow{2}{*}{ Material } & $\begin{array}{c}\sigma_{y} \\
\mathrm{MPa}\end{array}$ & $\begin{array}{c}\sigma_{y}^{c} \\
\mathrm{MPa}\end{array}$ & $\begin{array}{c}\sigma_{u} \\
\mathrm{MPa}\end{array}$ & $\begin{array}{c}E \\
\mathrm{GPa}\end{array}$ & $\begin{array}{c}v_{e} \\
-\end{array}$ & $\begin{array}{c}d_{i} \\
\mathrm{~mm}\end{array}$ & $\begin{array}{c}d_{o} \\
\mathrm{~mm}\end{array}$ \\
\hline PA38-T6 & 191 & 206 & 229 & 68.3 & 0.34 & 10 & 13 \\
E235 & 255 & 242 & 375 & 196.4 & 0.32 & 8 & 11 \\
E355 & 380 & 285 & 473 & 208.6 & 0.32 & 8 & 11 \\
1.4301 & 265 & 296 & 649 & 200.8 & 0.32 & 7 & 9.7 \\
\hline
\end{tabular}

All tests were performed on an Instron 8874 axial-torsional closed-loop servo-hydraulic testing system. Fatigue tests were performed in strain-controlled mode and axial and shear strains were measured by an Epsilon 3550 biaxial extensometer with a gauge length equal to $10 \mathrm{~mm}$ and measuring normal $\varepsilon= \pm 1 \mathrm{~mm} / 10 \mathrm{~mm}= \pm 10 \%$ and shear $\gamma= \pm 2^{\circ}$ strain ranges.

In the case of fully reversed sine-shaped loadings, which were applied in the experimental part of this study, the waveforms of those strains can be written as:

$$
\begin{gathered}
\varepsilon(t)=\varepsilon_{a} \sin \left(2 \pi f_{\varepsilon} t\right) \\
\gamma(t)=\gamma_{a} \sin \left(2 \pi f_{\gamma} t+\delta\right)
\end{gathered}
$$

where $\varepsilon_{a}, \gamma_{a}, f_{\varepsilon}, f_{\gamma}$ are amplitudes and frequencies of normal and shear strain, respectively, and $\delta$ is a phase shift angle. A proportional loading takes place when $\delta=0$ and $f_{\gamma} / f_{\varepsilon}=1$. If $\delta \neq 0$ and $f_{\gamma} / f_{\varepsilon}=1$ such a loading case is non-proportional, and it's called an out-ofphase loading. As much as 9 different loading cases were selected, but not all of them were utilized in case of each material. Details are given in Table 1. Parameters of selected asynchronous loadings are presented in Fig. 1. The selection of parameters of asynchronous loads, in particular, the ratio of amplitudes $\lambda$ and the ratio of frequencies $f_{\gamma} / f_{\varepsilon}$ was made in such a way that the share of normal and shear components within a given loading case was various. This share regards a different number of cycles of strain components and value of external forces work.

Table 1. Asynchronous loadings applied for individual materials.

\begin{tabular}{|c|c|c|c|c|c|}
\hline Material & \multicolumn{5}{|c|}{ Applied asynchronous loadings } \\
\hline PA38 & ASN1 & ASN2a & ASN3a & ASN4c & ASN5 \\
\hline E235 & ASN1 & ASN2a & ASN3b & ASN4a & \\
\hline E355 & ASN1 & ASN2b & ASN3b & ASN4b & ASN5 \\
\hline 1.4301 & ASN1 & ASN2a & ASN3a & ASN4b & ASN5 \\
\hline
\end{tabular}



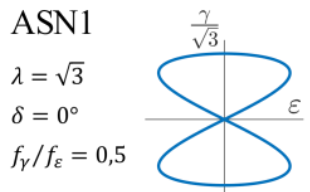

ASN3a

$\lambda=2 \sqrt{3}$

$\delta=0^{\circ}$

$f_{\gamma} / f_{\varepsilon}=0,2$

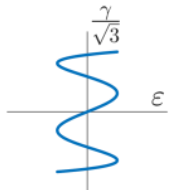

$\mathrm{ASN} 4 \mathrm{~b}$

$\lambda=3 \sqrt{3} / 5$

$\delta=0^{\circ}$

$f_{\gamma} / f_{\varepsilon}=6$

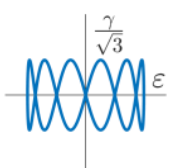

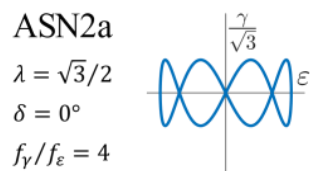

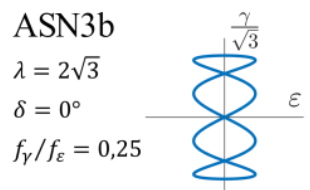

ASN4c

$\lambda=\sqrt{3}$

$\delta=0^{\circ}$

$f_{\gamma} / f_{\varepsilon}=8$

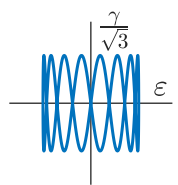

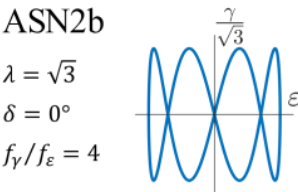

ASN4a

$\lambda=\sqrt{3} / 2$

$\delta=0^{\circ}$

$f_{\gamma} / f_{\varepsilon}=2 / 3$

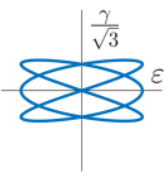

ASN5

$\lambda=\sqrt{3}$

$\delta=0^{\circ}$

$f_{\gamma} / f_{\varepsilon}=0,7$

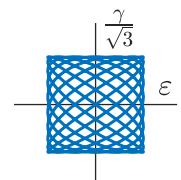

Fig. 1. Strain paths, waveforms and parameters of applied asynchronous loadings.

\section{Results}

\subsection{Cyclic stress-strain behaviour}

For the cyclic stress-strain behaviour analysis midlife hysteresis loops were recorded. In the case of asynchronous loadings, there are more than one hysteresis loop for one or both channels (normal or shear), recorded in one common period (block of loading). When the one common period equals the period of the strain component of lower frequency, the shape of hysteresis loops for this channel is complex. There are many local extrema of stress. Consequently, there are no tips in case of such hysteresis loops. For the second channel, there are few hysteresis loops.
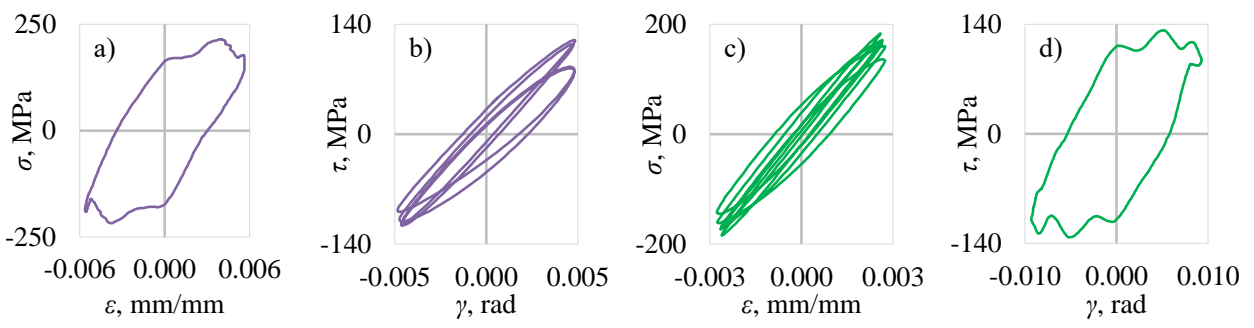

Fig. 2. Mid-life strain hysteresis loops recorded for PA38-T6 aluminium alloy: a) ASN2a ${ }^{\mathrm{N}}$

b) ASN2a $\mathrm{a}^{\mathrm{s}}$, c) ASN3 $\mathrm{a}^{\mathrm{N}}$, d) ASN3a $\mathrm{a}^{\mathrm{s}}$; superscripts: $\mathrm{S}$ - shear, $\mathrm{N}$ - normal.

In this study the maximum in time values of equivalent stress and strain were selected for comparison:

$$
\begin{gathered}
\sigma_{e q}=\max _{t}\left(\sqrt{\sigma(t)^{2}+3[\tau(t)]^{2}}\right) \\
\varepsilon_{e q}=\max _{t}\left(\frac{1}{\sqrt{2}\left(1+v_{e q}\right)} \sqrt{\varepsilon(t)^{2}\left(1+v_{e q}\right)^{2}+\frac{3}{2}[\gamma(t)]^{2}}\right)
\end{gathered}
$$


where equivalent Poisson's ratio $v_{e q}[6,7]$.

A comparison of cyclic stress-strain data is presented in Fig. 3. For PA38-T6 aluminium alloy small, but evident uniaxial cyclic hardening can be observed (Fig. 3.a). For E235 and E355 steels, uniaxial cyclic hardening cannot be unambiguously identified since these materials exhibited yield point elongation in a strain range tested under fatigue loadings. In the case of E235, most of the points corresponding to TC loading are located above the upper yield stress, while in the case of E355 steel all of them are below this value. For 1.4301 steel a relatively small uniaxial cyclic hardening was noticed. However, the difference between the monotonic stress-strain curve and points for TC loading increased with the increase of applied strain amplitude.

For PA38-T6 aluminium alloy, E235 and E355 steels equivalent stresses determined for TOR loading were noticeably higher than for TC loading and stresses under IP loading were very similar to those observed for TOR loading. In the case of 1.4301 equivalent stress values for TC, TOR and IP loadings were quite similar. For this material some scatter of points can be noticed. It is a result of relatively unstable and different equivalent stress histories recorded for mentioned loadings.

All tested materials shown additional non-proportional hardening under OP loading as well as under all of the applied asynchronous loadings. Some interesting observations can be made, based on the obtained data. It was noticed that non-proportional hardening isn't the same for all equivalent strain ranges. The amount of non-proportional hardening, as well as its relation to equivalent strain level, differs between applied cases of non-proportional loadings. They are both path- and material-depended.
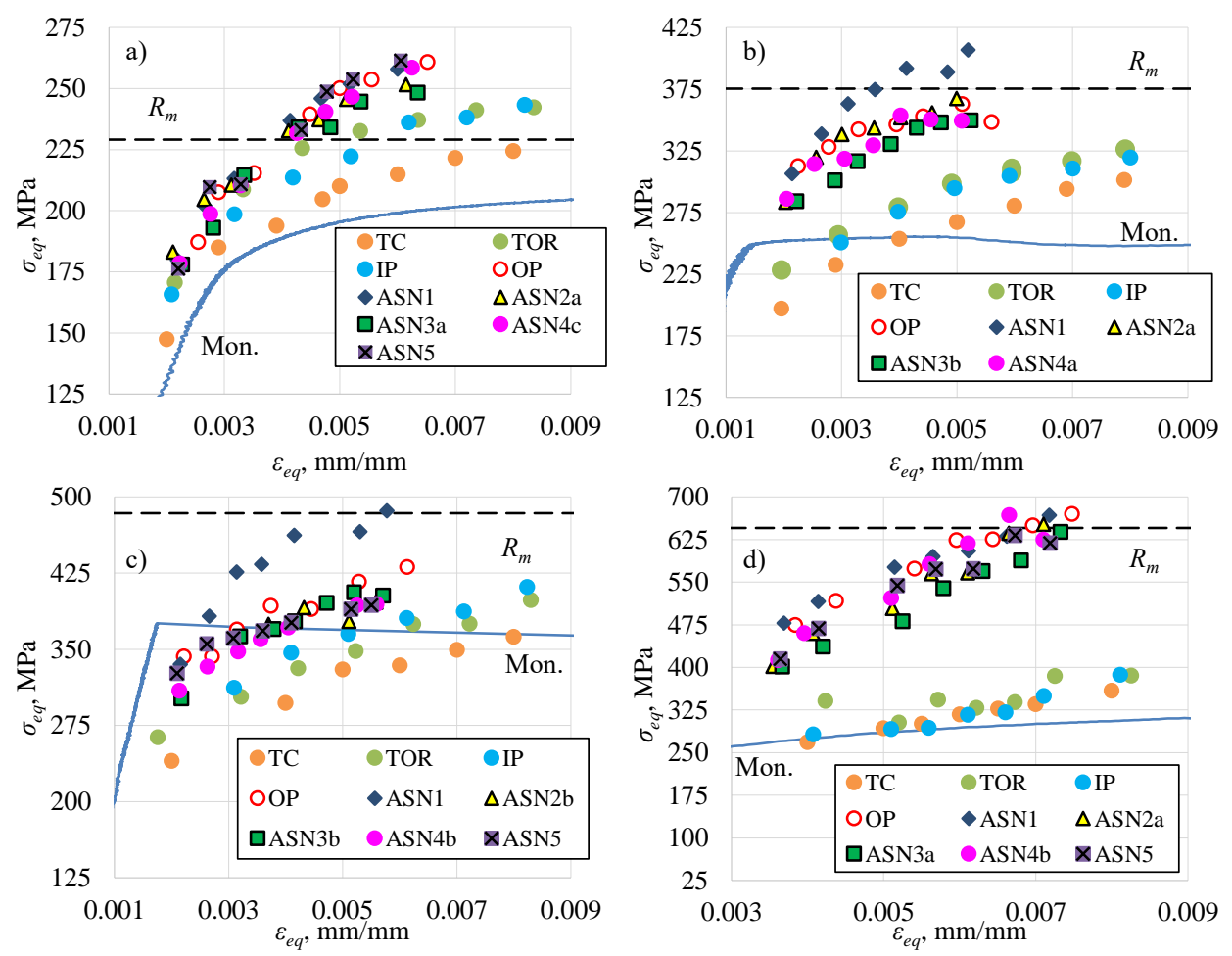

Fig. 3. A comparison of mid-life cyclic Huber-Mises equivalent stress-strain data and superimposed monotonic data for a) PA38-T6 aluminium alloy, b) E235 steel, c) E355 steel, d) 1.4301 steel. 


\subsection{Fatigue lives}

The assumption of this study was to presents results of fatigue life testing in a raw and simple form. Thus, obtained fatigue lives were shown in charts of equivalent Huber-Mises strain (equation (2)) versus a number of reversals to failure. The failure of the specimen was defined as a rapid change in the value of equivalent stress or approximately $90 \%$ drop. It corresponded to the presence of a few millimetres long crack or total separation of the specimen in few cases. Bearing in mind a discussion on loading's cycle definition regarding asynchronous loadings provided above, two different ways of results interpretation were used. In the first one the number of reversals $2 N_{f}{ }^{I}$ is equal to the number of reversals of strain component of lower frequency. For all asynchronous loadings, except ASN4a and ASN5, $2 N_{f}^{I}$ is equal to double the number of loading path (block) repetitions. This in turn corresponds to the approach most commonly found in the literature. In a second method the number of reversals $2 N_{f}{ }^{I I}$ is equal to the number of reversals of strain component of higher frequency.

In Fig. 4 results of fatigue lives testing were presented for TC, TOR and IP loadings, which are proportional. In the case of PA38-T6 aluminium alloy, E235 and E355 steels equivalent strain correlates relatively well with fatigue lives. It means that for those materials the differences in fatigue lives for IP and non-proportional loadings can be clearly interpreted as the effect of non-proportionality. For 1.4301 steel a high discrepancy between lives for TC and TOR loadings can be observed. Thus, the fatigue lives for non-proportional loadings are both influenced by the effect of non-proportionality and shear to normal strain ratio in the adopted coordinates system.
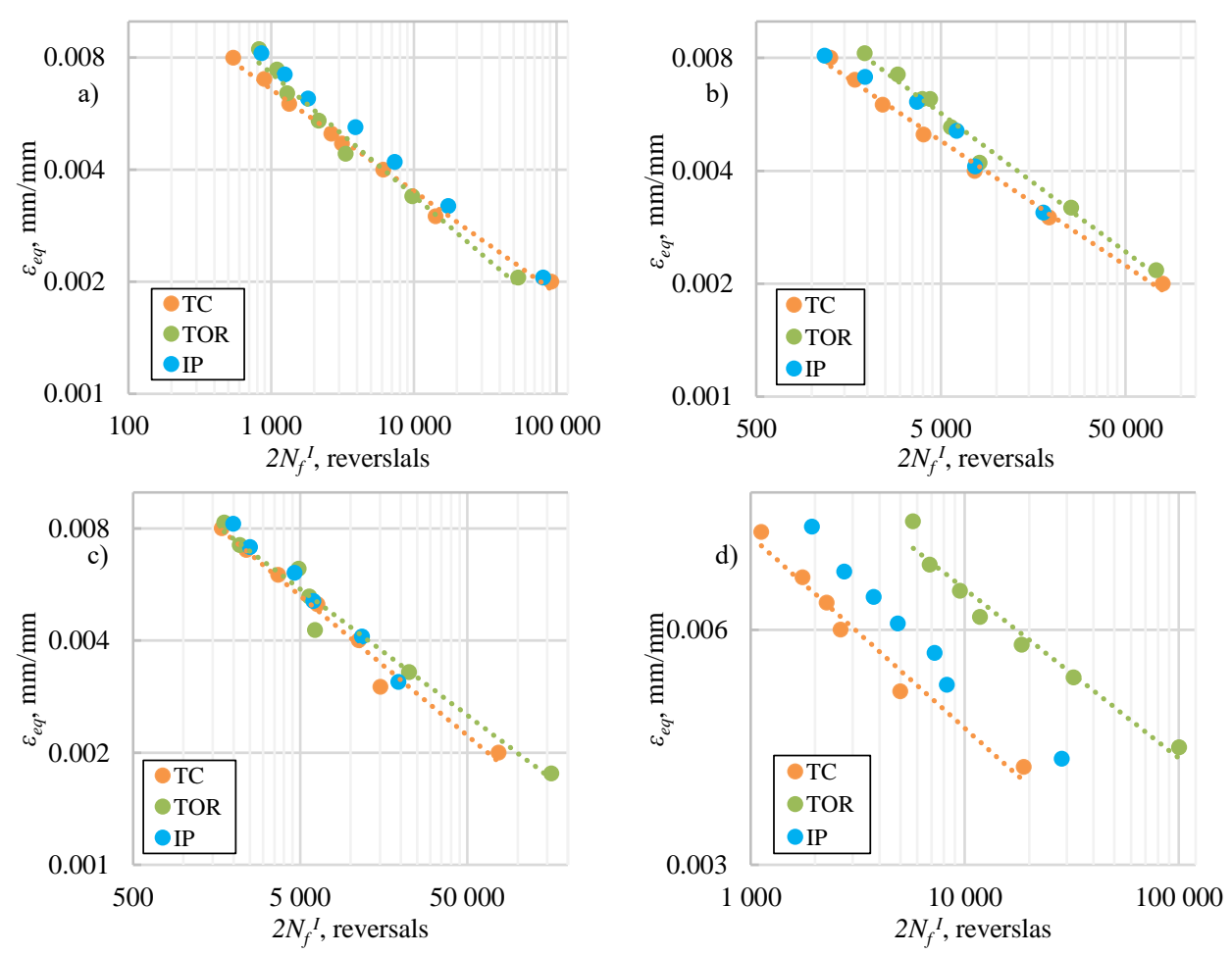

Fig. 4. Fatigue lives of a) PA38-T6 aluminium alloy, b) E235 steel, c) E355 steel and d) 1.4301 steel for axial, torsional and in-phase loadings. 
Fatigue loadings for IP and all non-proportional loadings are presented in Fig. 5. For PA38-T6 aluminium alloy curves fitted for IP and OP loadings have noticeably different slopes. The difference in fatigue lives is higher for high levels of equivalent strain and lower for low levels. Similar behaviour observed for ASTM A-516 Gr. 70 steel was interpreted by Ellyin et al. as a decrease in out-of-phase loading effect, accompanying the decrease of plastic strain [3]. Fatigue lives for ASN1 loading are the shortest if $2 N_{f}^{I}$ reversal definition is used. The best fit curve has a significantly different slope than in case of OP loading and it's approximately parallel to the curve for IP loading. If $2 N_{f}{ }^{I I}$ was applied, the fatigue lives for ASN1 and OP loadings were similar for high levels of equivalent strain and shorter for ASN1 loading on lower levels of strain. Data point for other asynchronous loadings were not fitted in the present with curves for the sake of clarity. However, a detailed observation would show that data points have similar trends for IP, ASN1, ASN2a, ASN3a loadings and OP, ASN4c, ASN5 loadings. Yet, no relationship between $\lambda, f_{\gamma} / f_{\varepsilon}$ or shear to normal strain energy ratio and those trends was found. If fatigue lives were compared using $2 N_{f}{ }^{I}$, the data points for all asynchronous loadings are placed between curves fitted for ASN1 and IP loadings. When results interpretation is performed using $2 N_{f}{ }^{I I}$, the data points for all asynchronous loadings, except ASN1, are located relatively close to the curve fitted for IP loading. For some of them, fatigue lives are shorter while for others longer than for IP loading, depending on loading case and level. The increase in fatigue lives when compared to $2{N_{f}}^{I}$ isn't however simple the result of $f_{\gamma} / f_{\varepsilon}$ ratio. For E235 steel the curves fitted for OP and ASN1 loadings also have different slopes. The curve for OP loading is approximately parallel to the one fitted for IP loading and the curve for ASN1 loading approaches it slightly for low levels of equivalent strain. When $2 N_{f}^{I}$ reversal definition is applied, the data points for all non-proportional loadings are approximately arranged into one common band. The highest discrepancy can be observed for the lowest loading level. If $2 N_{f}^{I I}$ is used, the fatigue lives for ASN2a and ASN3b are slightly lower than for IP loading for higher levels of equivalent strain and approximately equal for lower levels. The fatigue lives for ASN1 and ASN4a loadings are very similar. They are longer than for OP loading, but important reduction comparing to IP loading can be observed. In the case of E355 steel, the trends of data points for IP, OP and ASN1 are in a relation analogous to that observed in the case of E235 steel. Fatigue lives for $\mathrm{OP}, \mathrm{ASN} 1$ and ASN2b are quite similar if $2 N_{f}{ }^{I}$ is used. For ASN3b and ASN5 loadings they are slightly longer. All non-proportional loadings are characterized by fatigue lives significantly shorter comparing to IP loading. When $2 N_{f}{ }^{I I}$ is applied, fatigue lives for OP loading are noticeably shorter than for asynchronous loadings. Among them, fatigue lives for ASN1 are shortest. Decrease in fatigue life can be observed also for ASN5 and ASN2b loadings. For ASN3b and ASN4b loadings, fatigue lives are similar to those for IP loading. For 1.4301 steel the fatigue lives for OP and ASN1 loadings are almost identical if $2 N_{f}^{I}$ the definition is used, and both are much shorter than in case of IP loading. Data points for the rest of applied asynchronous loadings, ASN2a, ASN3a, ASN4b and ASN5 are placed between IP and OP loadings' best fit curves. For those loadings, fatigue lives are quite similar at high levels of strain and the scatter increases with the decrease of equivalent strain level. When the results are compared using $2 N_{f}{ }^{I I}$, the fatigue lives are the shortest for OP loading. For ASN1 and ASN5 loadings, fatigue lives are shorter than in case of IP loading. For ASN2a loading fatigue lives are approximately the same as in case of IP loading while for ASN3a and ASN4b they are longer. 

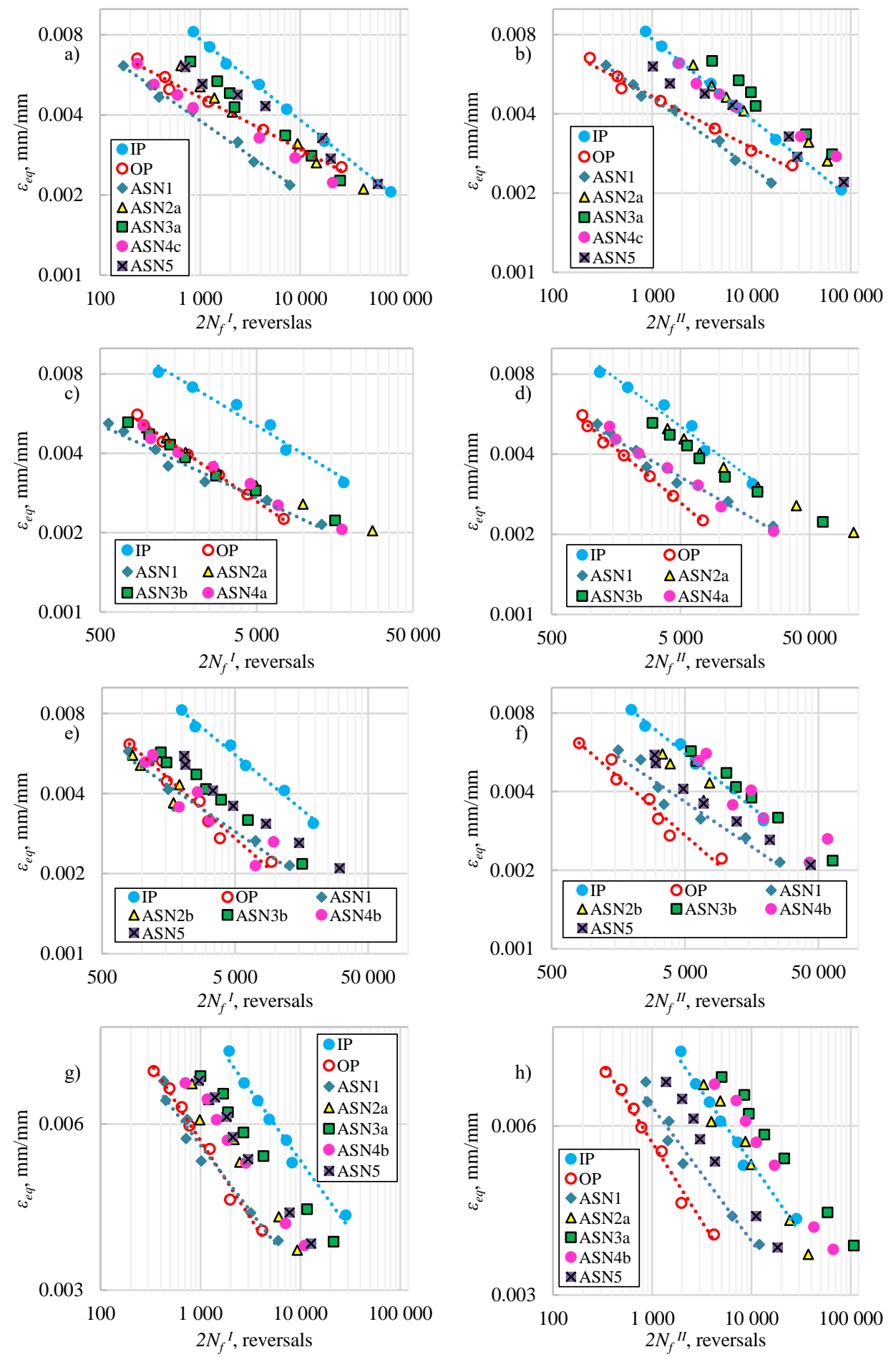

Fig. 5. Fatigue lives of PA38-T6 aluminium alloy for multiaxial loadings using a) $2 N_{f}{ }^{I}$ and b) $2 N_{f}{ }^{I I}$ reversal definition, E235 steel using c) $2 N_{f}^{I}$ and d) $2 N_{f}^{I I}$ reversal definition, E355 steel using e) $2 N_{f}{ }^{I}$ and f) $2 N_{f}^{I I}$ reversal definition, 1.4301 steel using g) $2 N_{f}^{I}$ and h) $2 N_{f}^{I I}$ reversal definition. 


\section{Discussion and conclusions}

1) Asynchronous loadings have a strong impact on stress-strain response. There is a various number of shear and normal strain hysteresis loops for one closed loading path (loading block) and they have a complex shape.

2) Under asynchronous loadings significant non-proportional hardening occurred, comparing to $90^{\circ}$ out-of-phase loadings. Thus, asynchronous loadings should be considered as strongly non-proportional in view of stress-strain response. The amount of non-proportional hardening was both path- and material-dependent.

3) Considering fatigue lives under proportional loadings it should be noted that 1.4301 steel had much different ratio of fatigue strength under axial and torsional lading than other tested materials.

4) In consequence, the Huber-Mises equivalent strain was sufficient to correlate fatigue lives for proportional loadings in case of PA38-T6 aluminium alloy and E235 and E355 steels, but not in case of 1.4301. It also fails as a damage parameter for non-proportional loadings, since the fatigue curves for proportional and non-proportional loadings do not coincide.

5) For all tested materials a significant reduction of fatigue life was observed under OP loading, comparing to IP loading.

6) Two elemental definitions of asynchronous loading cycle were applied for a simple presentation of results, denoted as $2 N_{f}^{I}$ and $2 N_{f}^{I I}$. If the first one was used, all asynchronous loadings cases resulted in a reduction of fatigue life, comparing to proportional loadings. In turn, when the second definition was used some cases of asynchronous loadings resulted in a reduction of fatigue life while others in an increase of fatigue lives.

7) Performed analyses showed that in case of asynchronous loadings one simple definition of loading cycle cannot be used. For the purpose of fatigue life estimation there seem to be two possible solutions:

- the asynchronous loading path can be treated as a block of loading and cycle counting appropriate for selected fatigue damage parameter should be performed, or

- the asynchronous loading path can be treated as one cycle, but associated fatigue damage parameter must take into account the whole loading history, in other words, it cannot be based only on strain and stress ranges.

8) It was shown that asynchronous loadings are clearly non-proportional, but more complex than out-of-phase loadings at the same time. Thus, experimental results obtained using asynchronous loadings can be an interesting and challenging data set for the purpose of fatigue damage models evaluation.

\section{References}

1. D. Socie, G. Marquis. Multiaxial Fatigue. SAE International, United States (2000).

2. A. Fatemi, N. Shamsaei, Multiaxial fatigue: An overview and some approximation models for life estimation. Int. J. Fatigue. 33: 948-958 (2011).

3. F. Ellyin, K. Gołoś, Z. Xia. In Phase and Out-of-Phase Multiaxial Fatigue. J. Eng. Mater. Technol 113: 112-118 (1991).

4. J. Mei, P. Dong. A new path-dependent fatigue damage model for non-proportional multi-axial loading. Int. J. Fatigue. 90: 210-221 (2016).

5. D. Skibicki, Ł. Pejkowski. The relationship between additional non-proportional hardening coefficient and fatigue life. Int. J. Fatigue. 123: 66-78 (2019). 
6. N. Shamsaei, A. Fatemi, D.F. Socie. Multiaxial fatigue evaluation using discriminating strain paths. Int. J. Fatigue. 33: 597-609 (2011).

7. B. Zhong, Y. Wang, D. Wei, K. Zhang, J. Wang. Multiaxial fatigue life prediction for powder metallurgy superalloy FGH96 based on stress gradient effect. Int. J. Fatigue. 109: 26-36 (2018). 